\title{
Single-nucleotide polymorphisms: analysis by mass spectrometry
}

\author{
Sascha Sauer ${ }^{1}$, Richard Reinhardt ${ }^{1}$, Hans Lehrach ${ }^{1}$ \& Ivo G Gut ${ }^{2}$ \\ ${ }^{1}$ Max Planck Institute for Molecular Genetics, Department of Vertebrate Genomics, Ihnestrasse 63-73, 14195 Berlin-Dahlem, Germany. ${ }^{2}$ Centre National de Génotypage, \\ Bâtiment G2, 2 Rue Gaston Crémieux, 91057 Evry Cedex, France. Correspondence should be addressed to S.S. (sauer@molgen.mpg.de).
}

Published online 22 November 2006; doi:10.1038/nprot.2006.257

\begin{abstract}
Matrix-assisted laser desorption-ionization (MALDI) mass spectrometry has evolved as a powerful method for analyzing nucleic acids. Here we provide protocols for genotyping single-nucleotide polymorphisms (SNPs) by MALDI based on PCR and primer extension to generate allele-specific products. Furthermore, we present three different approaches for sample preparation of primer-extension products before MALDI analysis and discuss their potential areas of application. The first approach, the 'GOOD' assay, is a purificationfree procedure that uses DNA-modification chemistry, including alkylation of phosphorothioate linkages in the extension primers. The other two approaches use either solid-phase extraction or microarray purification for the purification of primer-extension products. Depending on the reaction steps of the various approaches, the protocols take about 6-8 hours.
\end{abstract}

\section{INTRODUCTION}

Matrix-assisted laser desorption-ionization (MALDI) mass spectrometry has become an important, versatile tool in life science ${ }^{1,2}$ (Fig. 1). Mass spectrometry detects the mass/charge ratio $\left(\mathrm{m} \mathrm{z}^{-1}\right)$ of analyte ions in positive-ion mode or in negative-ion mode and produces highly accurate data ${ }^{1,3}$. MALDI mass spectrometry is efficiently used for quality control of oligonucleotides and for the analysis of genetic markers such as single-nucleotide polymorphisms (SNPs) ${ }^{4}$. MALDI-based SNP-genotyping procedures can be easily extended to the detection of cytosine methylation in genomic DNA as well as to the analysis of allele-specific expression and detection of alternative splicing ${ }^{3}$. In genotyping, mass spectrometry offers a distinct advantage over fluorescence-based methods in that the unambiguous determination of marker alleles relies on the direct measurement of the molecular weight of allele-specific products. Before MALDI, the products of SNPs are generated by a molecular biology procedure consisting of PCR amplification of the SNP positions of interest, digestion of residual dNTPs by phosphatase treatment, and an allele-specific primer-extension reaction ${ }^{5,6}$.

Because experimental molecular biology reactions are done in buffered solutions that contain large amounts of salts, it is necessary to include a step to eliminate those from the sample preparation. Phosphate residues of nucleic acids are a problem for MALDI detection because they provide a site of negative charge in solution. In particular, alkali metal ions such as sodium and potassium interfere with the ionization process, which weakens the analysis. Moreover, chemicals such as urea or guanidine- $\mathrm{HCl}$ and detergents perturb matrix crystallization and decrease signals in MALDI. Suitable preparation procedure steps must be taken before MALDI to circumvent those problems and to obtain high-quality mass spectra.

Many procedures for the detection of SNPs have been developed. The mass spectrometry companies Sequenom and Bruker Daltonics offer products tailored to SNP 'typing' applications. More information about their respective protocols is available on the Sequenom and Bruker Daltonics websites. Here we focus on our own developments, which can be easily used and adopted with a high degree of independency and flexibility.

We present protocols for the generation of products of the SNP rs607759 (dbSNP accession number; http://www.ncbi.nlm.nih.gov/
projects/SNP) based on primer extension (Fig. 1) as an example of these procedures. Moreover, we provide three different ways of preparing these products of SNPs for MALDI analysis. Each preparation procedure offers specific advantages depending on the application (Table 1).

The first, the GOOD assay (Fig. 1, A), is based on chemical DNA modification ${ }^{7-9}$. In this method, no stringent purification is required, leading to a high-throughput and cost-efficient SNP-genotyping procedure, particularly for studies of candidate genes. The second approach (Fig. 1, B) uses reverse-phase purification in 96-well plate format ${ }^{10}$, which is more expensive than the GOOD assay. However, this shorter, robust method can be applied easily to medium-throughput diagnostic applications and is particularly useful for researchers new to mass spectrometry technology. The third approach (Fig. 1, C) uses a new microarray format that allows sample purification and detection on the same device ${ }^{11}$. This procedure represents an emerging technology that (still) requires specific equipment, such as nanodispensing robots. It combines some of the advantages of parallel microarray 'readout' and highly accurate MALDI detection. In the following sections we comment on the different steps of our SNP-genotyping procedures and provide information about potential extension of these procedures for further applications.

\section{PCR}

PCR is used to amplify an SNP of interest and to reduce the complexity of the genome. Mass spectrometry offers flexibility in primer design. The user designs PCR primers by applying software available on the internet. We recommend the software Primer3 (http://frodo.wi.mit.edu/cgi-bin/primer3/primer3.cgi) for primer design. In our experience, a wide range of PCR fragment sizes can be used, from about $100 \mathrm{bp}$ up to $1,500 \mathrm{bp}$. We prefer single SNP analysis, because this approach produces high success rates without extensive optimization. The efficiency of the enzymatic reactions in a 'multiplex' assay (a combination of several loci or SNPs in a protocol) depends on the respective loci that must be combined, as the surrounding DNA sequences have an influence 
Figure 1 | General procedures for genotyping SNPS by MALDI mass spectrometry. These procedures include PCR of a genomic region containing SNPs, Antarctic phosphatase digestion of residual dNTPs of the PCR, and primer extension for the generation of allele-specific products of SNPs. For the GOOD assay (A), the primer-extension (PE) products are transformed in two steps into chargetagged nucleic acids, carrying one negative 'fix' charge, thus eliminating the need for purification before MALDI analysis. First, the primer-extension product is digested by phosphodiesterase II; this process is inhibited by phosphorothioate linkages in the primer. Second, the negatively charged phosphorothioate linkages are neutralized in the alkylation reaction. The phosphate linkage remains negatively charged. Because $\alpha$-cyano-4hydroxycinnamic acid methyl ester is used as the matrix, nucleic acids 'tagged' with a negative charge can be detected with high efficiency in the negative-ion mode of the mass spectrometer but not in the positive-ion mode (additional details, ref. 9). The second procedure (B) uses simple solid-phase extraction by 0asis, consisting of the loading of primer-extension products onto the reverse-phase resins, washing and elution before conventional preparation on a MALDI target plate. The third approach (C) applies new microarray devices that can be used for the purification and detection of DNA products. Primer-extension samples are spotted onto the device, which is then dipped into water to purify the samples of salts and detergents. Finally, the matrix is spotted onto the samples for direct MALDI detection. Unmodified nucleic acids such as the products of procedures $B$ and $C$ can be detected in the positive-ion and negative-ion detection modes with 3-hydroxypicolinic acid as the matrix. In our experience, analysis in the positive-ion detection mode generates slightly better results.

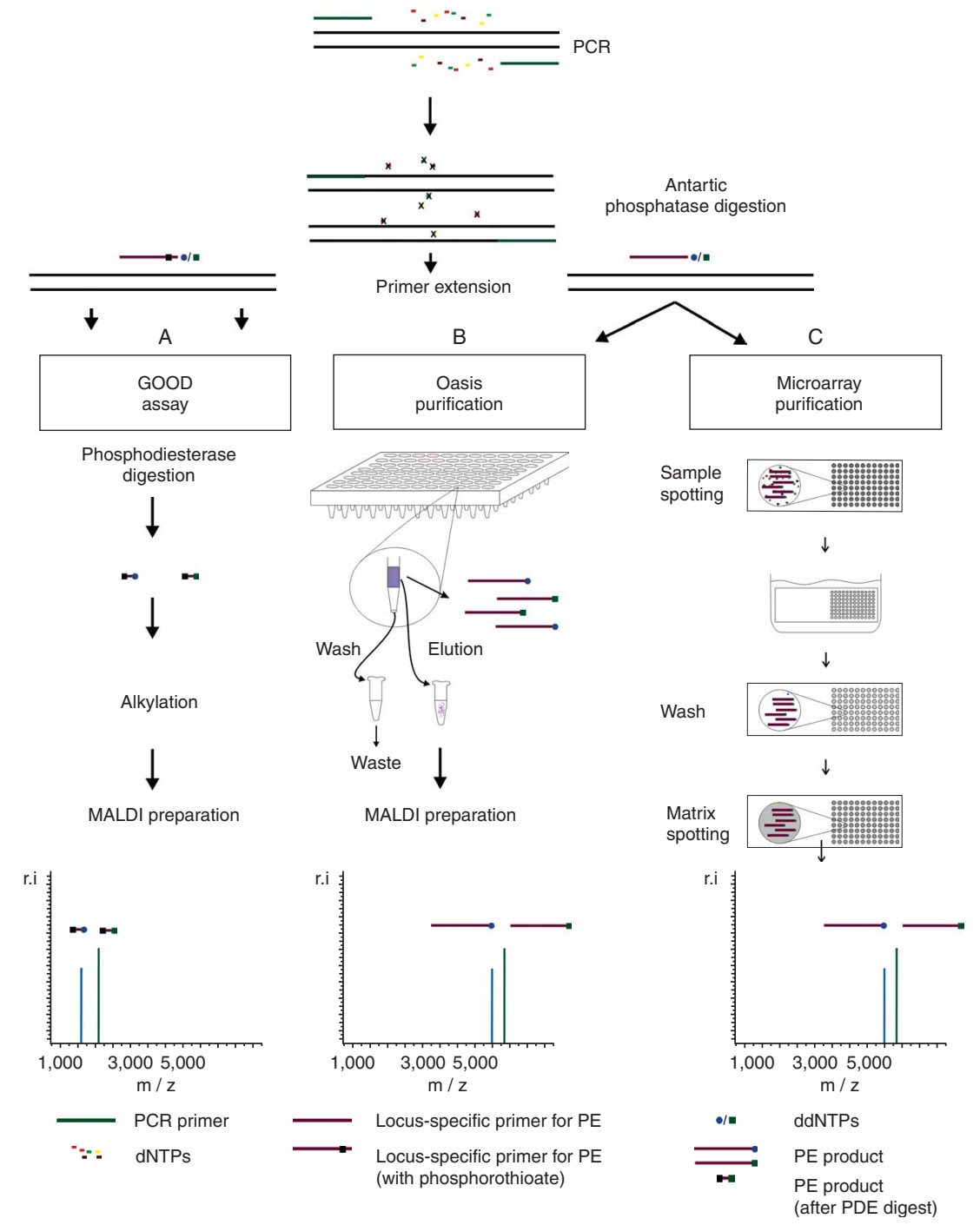

on the stability of the assay. The template can be any nucleic acid that can be amplified by PCR. For SNP genotyping, that is mostly purified genomic DNA, but crude samples such as tissue samples digested with proteinase $\mathrm{K}$ can also be used ${ }^{8}$. In general, the efficiency of SNP-genotyping experiments depends very much on the quality and integrity of the template DNA. In most cases, genomic DNA is purified from blood with commercially available kits. Whole-genome amplified DNA produced using suitable kits (e.g., AmpliPhi from Amersham) can also be used as template DNA for genotyping. SNP-genotyping methods based on PCR can be extended to the detection of DNA methylation with bisulfite-treated DNA ${ }^{12}$. Moreover, with single molecules $^{13}$ or cloned fragments of genomic DNA ${ }^{14}$, the SNP genotyping method can be used for molecular haplotyping.
A further strategy for molecular haplotyping consists of applying allele-specific PCR ${ }^{15}$.

\section{Antarctic phosphatase digestion}

We digest samples using Antarctic phosphatase (New England Biolabs) to hydrolyze residual dNTPs of the PCR, which could interfere with the subsequent primer-extension reaction. This enzyme catalyzes the removal of $5^{\prime}$ phosphate groups from nucleotides, DNA and RNA with higher efficiency than shrimp alkaline phosphatase, which has been used in published protocols ${ }^{7-9}$.

\section{Primer extension}

The primer-extension reaction is used to generate allele-specific products. A primer is chosen upstream of the SNP to be genotyped.

TABLE 1 | Main advantages and disadvantages of the three approaches.

\begin{tabular}{|c|c|c|}
\hline Assay & Advantages & Disadvantages \\
\hline (A) G00D assay & $\begin{array}{l}\text { Cheap; no purification; suitable for medium- to high-throughput } \\
\text { application }\end{array}$ & Use of highly toxic reagents; many steps \\
\hline (B) $0 \mathrm{as}$ & Easy protocol; suitable for diagnostic application & Expe \\
\hline
\end{tabular}


Primers can be placed on either strand of the PCR product and can contain phosphorothioate linkages (Fig. 1, A) or can be unmodified (Fig. 1, B and C). As with PCR, the user designs extension primers with software available on the internet. It is possible to use extension primers when there are adjacent SNPs, with the caveat that the four nucleotides upstream of the SNP must be devoid of SNPs. Primers for the extension reaction can be optimized with the appropriate software (such as the OLIGO v. 6.0 program). As with the PCR, we prefer single reactions, because this approach produces a high success rate without extensive optimization. Also as with the PCR, the efficiency of 'multiplex' primer extension (with a combination of several SNPs) depends on the SNPs that must be combined. Moreover, multiple products can affect each other in the MALDI detection process.

In the GOOD assay primer extension (no subsequent purification; Fig. 1, A), the second and third nucleotides from the $3^{\prime}$ end of the primer are connected by a phosphorothioate linkage (Fig. 2). Additionally, a second phosphorothioate can be linked between the third and fourth nucleotides from the $3^{\prime}$ end of the primer. The linkage between the first and the second nucleotide from the $3^{\prime}$ end and all other DNA backbone linkages, however, consists of regular phosphate groups. The phosphorothioate groups fulfill two functions: they can be quantitatively 'charge-neutralized' by alkylation and they inhibit the complete digestion of the primer in the fourth step of the procedure. For the primer-extension reaction in the GOOD assay, primers are combined with a specifically selected set of $\alpha$-S-ddNTPs. Their addition to the primer results in the formation of a further phosphorothioate linkage. For primer extension using unmodified oligonucleotides (with subsequent purification; Fig. 1, B and C), we use unmodified primers and a mixture of dNTPs and ddNTPs to generate allele-specific products of SNPs, so we must purify the respective products because of the interaction with salts and detergents in the MALDI process mentioned above.

\section{Phosphodiesterase II digestion (GOOD assay; Fig. 1, A)}

The step of phosphodiesterase II digestion is an essential part of the GOOD assay. Much of the primer is removed. The molecular

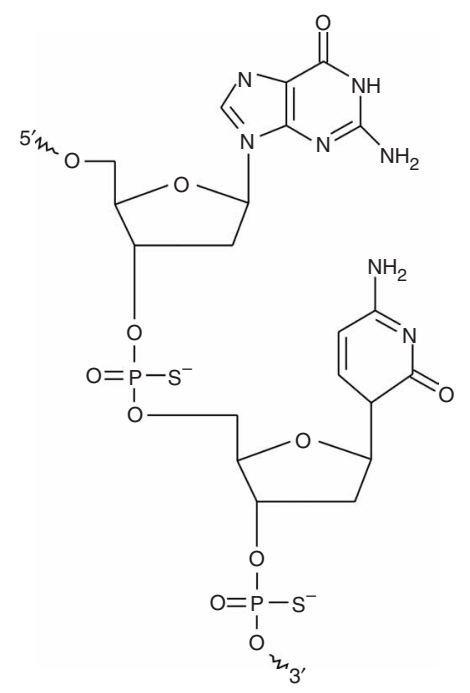

Figure 2 | Chemical structure of a phosphorothioate linkage in DNA. weights of the products are shifted into a mass range in which the detection sensitivity and resolution of the mass spectrometer are best. The digestion is inhibited by the phosphorothioate linkage(s) close to the $3^{\prime}$ end of the extension primer. The use of 'photo-cleavable' primers is another approach that would circumvent this enzymatic step ${ }^{16}$. However, the use of primers with such linkers generating an abasic site requires extensive optimization.

\section{Alkylation (GOOD assay; Fig. 1, A)}

The fifth step of the GOOD assay is alkylation. The phosphorothioate linkages remaining from the previous step are 'chargeneutralized' by methylation with iodomethane. The reaction conditions are chosen so that the selectivity of the reaction is optimal for the addition of methyl groups to phosphorothioates. The protocol should not be modified, as that might lead to the creation of side products caused by over- or underalkylation of the phosphodiesterase II products, which might complicate the analysis of SNP genotypes. The addition of the methylation agent results in the generation of two phases. The DNA products are in the upper, aqueous phase, which is sampled and diluted in solution. The product masses are in an $M_{\mathrm{r}}$ range of 1,000-2,000. In principle, the use of extension primers containing charge-neutral DNA backbones such as methylphosphonates could supersede the alkylation step. However, in practice, using extension primers with such modifications requires extensive optimization ${ }^{17}$.

\section{Purification of unmodified oligonucleotides with Oasis microelution plates (Fig. 1, B)}

Oasis microelution plates consist of a copolymer of divinylbenzene and $N$-vinylpyrrolidone ${ }^{18}$. The hydrophobic divinylbenzene moiety provides reverse-phase interaction, whereas the more hydrophilic $N$-vinylpyrrolidone increases the sorbent water 'wettability'. In contrast to many other reverse-phase resins, purification is not affected by drying during extraction and the resins can be used several times. Another advantage of this copolymer is that the support has no silanol activity, which reduces cation-exchange retention, resulting in reduced secondary retention of alkali metal ions and their potential coelution.

Sample preparation of GOOD assay products and primerextension products purified by Oasis solid-phase extraction See Fig. 1, A and B

Prior to MALDI analysis, for GOOD assay products, we use thinlayer preparation, and for the purified DNA products, we use dried droplet preparation ${ }^{5}$.

For thin-layer preparations, a suitable matrix such as $\alpha$-cyano-4hydroxycinnamic acid methyl ester is applied to the MALDI target plate in volatile acetone. This solvent spreads and evaporates immediately, leaving a thin layer of small matrix crystals. The DNA sample is dispensed onto the thin layer in a solvent that does not dissolve the matrix, such as $40 \%$ acetonitrile. Small oligonucleotides of the GOOD assay are thus built into the surface of the matrix.

For conventional dried-droplet preparation, low microliter volumes of an aqueous matrix solution, such as 3-hydroxypicolinic acid in ammonium citrate, are mixed with a solution containing oligonucleotides on the MALDI target plate. As a result of the uneven height of the dried-droplet preparation, the mass 
calibration in the observation window of 5,000-8,000 $\mathrm{m} \mathrm{z}^{-1}$ can be unstable. MALDI detection is based on the determination of the time of flight of an ion. The variable height of the matrix preparation results in a shifting of the starting position, which affects the time of flight, leading to mass variation of a few Daltons. However, for genotyping, this is not a problem, as the mass differences of SNP products are in the $M_{\mathrm{r}}$ range of 300 . In contrast, the thin-layer preparation of the GOOD assay gives less spot-tospot variation and better mass accuracy and resolution because of the smaller products detected.

\section{Microarray purification of unmodified oligonucleotides and sample preparation on the same device (Fig. 1, C)}

This new MALDI procedure for SNP analysis allows efficient binding of DNA to positively charged surfaces during washing and subsequent MALDI detection of the primers on the same surface. We use microscope slides modified with gold and coated with polyamidoamine (PAMAM) 'starburst dendrimers'. These slides can be clamped into adapters that fit the ionization chambers of many MALDI mass spectrometers. The dried-droplet procedure with 3-hydroxypicolinic acid can be 'miniaturized' to low nanoliter volumes to improve mass detection over the conventional procedure described above.

\section{Reagents}

Not all the reagents and equipment are required for each of our SNPgenotyping procedures; check which are needed by reading the protocols first. We provide protocols for single analyses done manually. Depending on the number of DNA samples to be analyzed, the user should prepare 'master mixes' that can be dispensed by robots. Automation of our assays is feasible with many commercially available devices other than the instruments we use.

\section{MATERIALS}

\section{REAGENTS}

-10× PCR buffer for 'home-made' Taq polymerase: $200 \mathrm{mM}$ Tris- $\mathrm{HCl}$,

$160 \mathrm{mM}\left(\mathrm{NH}_{4}\right)_{2} \mathrm{SO}_{4}$ and $250 \mathrm{mM} \mathrm{KCl}$

$\cdot \mathrm{MgCl}_{2}$

- dNTPs (Roche)

- Unmodified oligonucleotides (forward and reverse PCR primers; MWG)

- Taq polymerase for PCR ('in-house')

-Human genomic DNA (purified 'in-house' from whole blood)

- Adhesive film (Tesa)

- Antarctic phosphatase (New England Biolabs)

- TermiPol DNA polymerase for primer extension (Solis Biodyne)

-Extension primer, modified (oligonucleotide containing phosphorothioates; Biotez)

- $\alpha$-S-ddNTPs (Biolog)

- Oligonucleotides containing methylphosphonates (Eurogentec)

- Extension primer, unmodified (MWG)

- ddNTPs (Roche)

- Phosphodiesterase II (Worthington)

- Acetic acid (HPLC-grade; Sigma-Aldrich, Merck, Fluka)

-Acetonitrile (HPLC-grade; Sigma-Aldrich, Merck, Fluka)! CAUTION This

harmful, very toxic reagent should be handled appropriately.

- Iodomethane (HPLC-grade; Sigma-Aldrich, Merck, Fluka) ! CAUTION This

harmful, very toxic reagent should be handled appropriately.

- $\alpha$-cyano-4-hydroxycinnamic acid methyl ester (Bruker Daltonics)

-Acetone (HPLC-grade; Sigma-Aldrich, Merck, Fluka)

- Oasis HLB microelution plates, including 96 Oasis resins (Waters)

- Methanol

- Plasticware (96- and 384-well polypropylene plates; Eppendorf)

-3-hydroxypicolinic acid (Bruker Daltonics)

-Ammonium citrate ( $>98 \%$; Sigma-Aldrich, Merck, Fluka)

-Washing solution: 30\% isopropanol (HPLC-grade; Sigma-Aldrich, Merck,

Fluka) and $1 \%$ acetic acid in double-distilled water $\left(\mathrm{ddH}_{2} \mathrm{O}\right)$

- Gold PAMAM dendrimer microscope slides with amino coating (Chimera Biotec)

\section{EQUIPMENT}

-PTC 200 thermocycler (MJ Research)

- MTP AnchorChip 400/384 TF and adapter (Bruker Daltonics)

- Biflex III time-of-flight mass spectrometer equipped with a Scout MTP ion source with delayed extraction (Bruker Daltonics)

- SciFlex arrayer (Scienion)

-XMass software

- Ultrasonic bath (Branson)

- Sciclone (Caliper Life Sciences)

- Multimek 96/384 (Beckman Coulter)

- Hydra 96 (Robbins)

- Cybiwell 96 (Cybio)

- Oasis HLB purification (Waters)

- Biorobot 9600 with vacuum station (Qiagen)
- Slide adapter (made in-house; similar products can be purchased from

Bruker Daltonics)

- GenoTools SNP manager software (Bruker Daltonics)

- Centrifuge 5810R (Eppendorf)

- SpeedVac Plus SC 210A (Savant)

\section{REAGENT SETUP}

Primers PCR primer sequences for SNP rs607759 are forward, 5'-AGCTCTA AAACATGGAAAGGAAA- $3^{\prime}$, and reverse, 5' -TCATGCAATGAAGGGGTCTT AT-3'. The modified extension primer for SNP rs607759 (the GOOD assay; Fig. $1, \mathrm{~A}$ ) is $5^{\prime}$-AATTGAATGGCTCTAGG $\mathrm{pt}_{\mathrm{AC}} \mathrm{A}-3^{\prime}$, where the subscripted ' $\mathrm{pt}$ ' indicates a phosphorothioate linkage. The unmodified extension primer used for the other approaches for SNP rs607759 (Fig. 1, B and C) is 5'-AATTGAAT GGCTCTAGGAC-3'.

DNA polymerase In general, every DNA polymerase system that generates sufficient amplification products with high specificity can be used. Problems might arise in the final mass spectrometry of GOOD assay products because of specific residual agents (e.g., betaine and DMSO) and some detergents (e.g., Tween 20). Researchers using these procedures might inform us about potential problems, particularly if new DNA polymerases are tested.

Oligonucleotides for mass spectrometer external calibration External calibration is sufficient to generate reliable mass spectrometry results with our SNPgenotyping procedures. For external calibration for the detection of GOOD assay products, we recommend using four-, five- and six-residue oligonucleotides containing methylphosphonate linkages and one phosphate linkage (and $\alpha$-cyano-4-hydroxycinnamic acid methyl ester as the matrix). For larger, unmodified primer extension products, in combination with 3-hydroxypicolinic acid as the matrix, at least three oligonucleotides with masses spanning the mass range of the products of interest should be used.

\section{EQUIPMENT SETUP}

Liquid-handling robots Although 'master mixes' are prepared by hand, all pipetting steps can be done by liquid-handling robots. In our laboratory, we use the robots in Table 2 . The high success rates of mass spectrometry SNPgenotyping methods depend on accurate pipetting, as these procedures require the successive addition of reagents, making them potentially vulnerable to errors and contamination.

Oasis purification We use 96-well plates packed with $5 \mathrm{mg}$ Oasis HLB resins in each well (Waters). The solid-phase extraction procedure can be done with the appropriate manifold kit for Oasis HLB purification from Waters or any other vacuum station such as the Biorobot 9600 (Qiagen), on which 96-well plates such as the Oasis plates fit. Adjust the vacuum to $507,957 \mathrm{kPa}$ per step. Mass spectrometry Mass spectra are recorded with a Bruker Biflex III time-offlight mass spectrometer. Alternative mass spectrometers such as the Autoflex or Ultraflex mass spectrometer (Bruker Daltonics) or other MALDI mass spectrometers can also be used for our applications, after optimization. For optimization of detection parameters with alternative instruments, we recommend using the standard oligonucleotides described above for external calibration. The Biflex III time-of-flight mass spectrometer is equipped with a Scout MTP ion source. Use either the MTP AnchorChip 400/384 TF targets 
TABLE 2 | Liquid-handling robots and 'arrayers' used for the different steps of the procedures.

\begin{tabular}{ll}
\hline Step & Suitable robot \\
\hline PCR & Multimek 96/384, Cybiwell 96 \\
Antarctic phosphatase digestion & Multimek 96/384, Cybiwell 96 \\
Primer extension & Multimek 96/384, Cybiwell 96 \\
Phosphodiesterase digestion & Multimek 96/384, Cybiwell 96 \\
Transfer of primer-extension products for Oasis purification & Cybiwell 96 \\
Transfer of primer-extension products for microarray purification & SciFlex arrayer \\
Alkylation & Hydra 96 (under a fume hood) \\
Transfer alkylation product & Hydra 96 \\
Preparation on MALDI target & Multimek 96/384, Cybiwell 96, Sciclone, SciFlex arrayer \\
\hline
\end{tabular}

or the microarray slides as target plates and appropriate adapters that fit into the Scout MTP ion source.

We use MTP AnchorChip 400/384 TF targets for analysis of samples by the GOOD assay as well as for Oasis-purified samples. These devices have 384 hydrophilic spots ( $400 \mu \mathrm{m}$ in diameter). We create a thin-layer preparation of matrix and sample for the GOOD assay and a dried-droplet preparation for the Oasis-purified samples.

\section{PROCEDURE}

\section{PCR TIMING 90-120 min}

1| Prepare the reaction mixture as follows:

\begin{tabular}{lr}
\hline Reagent & Volume \\
\hline $\mathrm{ddH}_{2} \mathrm{O}$ & $4.9 \mu \mathrm{l}$ \\
$10 \times \mathrm{PCR}$ buffer & $1 \mu \mathrm{l}$ \\
$50 \mathrm{mM} \mathrm{MgCl}$ & $0.4 \mu \mathrm{l}$ \\
$2 \mathrm{mM}$ dNTPs & $0.5 \mu \mathrm{l}$ \\
Forward primer $(5 \mu \mathrm{M})$ & $1 \mu \mathrm{l}$ \\
Reverse primer $(5 \mu \mathrm{M})$ & $1 \mu \mathrm{l}$ \\
Taq polymerase $\left(5 \mathrm{U} \mathrm{l}^{-1}\right)$ & $0.2 \mu \mathrm{l}$ \\
Human genomic DNA $\left(10{\left.\mathrm{ng} \mathrm{l}^{-1}\right)}^{\text {Hum }}\right.$ & $1 \mu \mathrm{l}$ \\
\hline
\end{tabular}

For microarray purification, we use gold PAMAM dendrimer microscope slides, clamped in an adapter made in-house (or, alternatively, from Bruker Daltonics), with amino coating and a variation of dried-droplet preparation of the DNA sample and matrix.

For analysis of mass spectra quality and determination of genotypes, we use the GenoTools SNP manager software ${ }^{19}$.

2| Transfer the reaction mixture to a thermocycler for PCR as described in Table 3.

$\triangle$ CRITICAL STEP Experimental steps involving heat incubation and thermocycling at temperatures of $37^{\circ} \mathrm{C}$ or higher require closed tubes or sealed-well plates with adhesive film to avoid evaporation.

3| Pipet $3 \mu \mathrm{l}$ of the PCR product into a tube or a 96- or 384-well plate for the next step of the assay. The remaining $7 \mu \mathrm{l}$ are stored and can be used for an additional assay or for confirmation of the PCR by agarose-gel electrophoresis.

$\triangle$ CRITICAL STEP As little as $100 \mathrm{fmol}$ to $1 \mathrm{pmol}$ of the PCR product should be sufficient for the subsequent steps described below.

PAUSE POINT PCR products can be stored at $-20^{\circ} \mathrm{C}$.

? TROUBLESHOOTING

\section{Antarctic phosphatase digestion $\bigcirc$ TIMING 45 min}

4| Prepare the reaction mixture as follows:

\begin{tabular}{lr}
\hline Reagent & Volume \\
\hline PCR product & $3 \mu \mathrm{l}$ \\
Antarctic phosphatase $\left(5{\left.\mathrm{U} \mu \mathrm{l}^{-1}\right)}^{5} \mathrm{mM}\right.$ Tris- $\mathrm{HCl}, \mathrm{pH} 8.0$ & $0.25 \mu \mathrm{l}$ \\
\hline
\end{tabular}

5| Transfer the reaction mixture to a thermocycler and treat as follows:

\begin{tabular}{lc}
\hline Cycle number & Temperature \\
\hline 1 & 30 min at $37{ }^{\circ} \mathrm{C}$ \\
2 & 10 min at $90{ }^{\circ} \mathrm{C}$ \\
\hline
\end{tabular}

PAUSE POINT Treated PCR product solutions can be stored at $-20{ }^{\circ} \mathrm{C}$. ? TROUBLESHOOTING 
PROTOCOL

TABLE 3 | PCR conditions for Step 2.

\begin{tabular}{lccc}
\hline Cycle number & Denaturation & Annealing & Polymerization \\
\hline 1 & $4 \min$ at $95{ }^{\circ} \mathrm{C}$ & - & - \\
$2-41$ & $15 \mathrm{~s}$ at $95{ }^{\circ} \mathrm{C}$ & $30 \mathrm{~s}$ at $56{ }^{\circ} \mathrm{C}$ & $60 \mathrm{~s}$ at $72{ }^{\circ} \mathrm{C}$ \\
42 & & 5 min at $72{ }^{\circ} \mathrm{C}$ & \\
\hline
\end{tabular}

\section{Primer extension $\bigcirc$ TIMING 90-120 min}

6| Primer extension can be done with primers containing phosphorothioate linkages (for the G00D assay; option A) or with unmodified primers (followed by 0asis or microarray purification; options $B$ and $C$ ).

(A) G00D assay (no subsequent purification; Fig. 1, A)

(i) Prepare the reaction mixture as follows:

\begin{tabular}{lr}
\hline Reagents & Quantity \\
\hline Antarctic phosphatase product & $5 \mu \mathrm{l}$ \\
ddH ${ }_{2} \mathrm{O}$ & $0.5 \mu \mathrm{l}$ \\
TermiPol $\left(5{\left.\mathrm{U} \mu \mathrm{l}^{-1}\right)}^{\mathrm{m}} \mathrm{mM} \mathrm{MgCl}\right.$ & $0.32 \mu \mathrm{l}$ \\
Extension primer $(10 \mu \mathrm{M})$ & $0.08 \mu \mathrm{l}$ \\
$\alpha$-S-ddCTP $(1 \mathrm{mM})$ & $0.5 \mu \mathrm{l}$ \\
$\alpha$-S-ddTTP $(1 \mathrm{mM})$ & $0.3 \mu \mathrm{l}$ \\
\hline
\end{tabular}

(ii) Transfer the reaction mixture to a thermocycler and proceed with 45 cycles of $10 \mathrm{~s}$ at $95{ }^{\circ} \mathrm{C}$ (denaturation), $30 \mathrm{~s}$ at $55^{\circ} \mathrm{C}$ (annealing) and $15 \mathrm{~s}$ at $72{ }^{\circ} \mathrm{C}$ (polymerization).

(B) Primer extension (with subsequent Oasis purification; Fig. 1, B)

(i) Prepare the reaction mixture as follows:

\begin{tabular}{lr}
\hline Reagent & Volume \\
\hline Antarctic phosphatase product & $5 \mu \mathrm{l}$ \\
ddH $_{2} \mathrm{O}$ & $0.5 \mu \mathrm{l}$ \\
TermiPol $\left(5{\left.\mathrm{U} \mathrm{L}^{-1}\right)}^{-1}\right.$ & $0.32 \mu \mathrm{l}$ \\
$50 \mathrm{mM} \mathrm{MgCl}$ & $0.08 \mu \mathrm{l}$ \\
Extension primer $(10 \mu \mathrm{M})$ & $0.5 \mu \mathrm{l}$ \\
ddCTP $(2 \mathrm{mM})$ & $0.125 \mu \mathrm{l}$ \\
ddGTP $(2 \mathrm{mM})$ & $0.125 \mu \mathrm{l}$ \\
dTTP $(2 \mathrm{mM})$ & $0.125 \mu \mathrm{l}$ \\
\hline
\end{tabular}

(ii) Transfer the reaction mixture to a thermocycler and proceed with 35 cycles of $10 \mathrm{~s}$ at $95{ }^{\circ} \mathrm{C}$ (denaturation), $30 \mathrm{~s}$ at $50{ }^{\circ} \mathrm{C}$ (annealing) and $15 \mathrm{~s}$ at $72{ }^{\circ} \mathrm{C}$ (polymerization).

PAUSE POINT Primer-extension products (each variant) can be stored at $-20{ }^{\circ} \mathrm{C}$ for a week.

$\triangle$ CRITICAL STEP Freezing and thawing of $\alpha$-S-ddNTP stock solutions used in Step $6 \mathrm{~A}(i)$ should be minimized to prevent potential degradation (oxidation) of these modified nucleotides

? TROUBLESHOOTING

(C) Primer extension (with subsequent microarray purification; Fig. 1, C)

(i) Proceed as described in Steps $6 \mathrm{~B}(\mathrm{i}-\mathrm{ii})$.

7| Continue procedure with the G0OD assay (A), 0asis purification (B) or microarray purification (C).

(A) G00D assay TIMING 175 min

(i) For phosphodiesterase II digestion, reconstitute the dry phosphodiesterase II (25 U) in $5 \mathrm{ml} \mathrm{ddH}_{2} \mathrm{O}$. Store the remaining enzyme at $-20{ }^{\circ} \mathrm{C}$.

(ii) Prepare the reaction mixture as follows, then transfer to a thermocycler or incubator and incubate for 90 min at $37^{\circ} \mathrm{C}$ :

\begin{tabular}{lr}
\hline Reagent & Volume \\
\hline Primer-extension product & $7 \mu \mathrm{l}$ \\
Acetic acid $(0.5 \mathrm{M})$ & $0.5 \mu \mathrm{l}$ \\
Phosphodiesterase II $\left(5 \mathrm{U} \mathrm{ml}^{-1}\right)$ & $1.5 \mu \mathrm{l}$ \\
\hline
\end{tabular}

$\triangle$ CRITICAL STEP Use the digested products immediately for the next step, because after $90 \mathrm{~min}$, the digestion might also affect the phosphorothioate linkage even at room temperature $\left(25^{\circ} \mathrm{C}\right)$.

? TROUBLESHOOTING 
(iii) For alkylation, prepare the reaction mixture as follows:

\begin{tabular}{lc}
\hline Reagent & Volume \\
\hline Phosphodiesterase product & $9 \mu \mathrm{l}$ \\
Acetonitrile & $12 \mu \mathrm{l}$ \\
$\mathrm{ddH}_{2} \mathrm{O}$ & $3 \mu \mathrm{l}$ \\
Iodomethane & $6 \mu \mathrm{l}$ \\
\hline
\end{tabular}

I CAUTION Very toxic reagents (iodomethane and acetonitrile) are used for the alkylation reaction, so particular care must be taken with this procedure. Alkylation reagents must be dispensed in a fume hood.

(iv) Transfer the reaction mixture to a thermocycler and proceed as follows:

\begin{tabular}{ll}
\hline Cycle number & Temperature \\
\hline 1 & 30 min at $40^{\circ} \mathrm{C}$ \\
2 & $4^{\circ} \mathrm{C}$ (hold at this temperature) \\
\hline
\end{tabular}

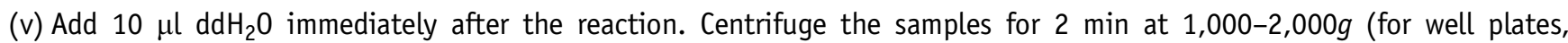
we use Centrifuge 5810R (Eppendorf)) or, alternatively, wait $10 \mathrm{~min}$ for phase separation. After the incubation, volatile iodomethane is in the lower phase of the reaction mixture, whereas the upper, aqueous phase contains the DNA products. Thus, evaporation of iodomethane is inhibited after the reaction has finished.

(vi) Prepare $10 \mu \mathrm{l}$ of $40 \%$ acetonitrile for mixing. Add $5 \mu \mathrm{l}$ of the upper phase of the alkylation reaction to $10 \mu \mathrm{l}$ of $40 \%$ acetonitrile.

$\triangle$ CRITICAL STEP For reaction mixtures, prepare at least twice as much 'master mix' as you actually need to add to the phosphodiesterase products. The 'master mix' will contain two phases; use $21 \mu \mathrm{l}$ of the lower phase. Alkylation and sample preparation of alkylated products must be done immediately. Final samples in $40 \%$ acetonitrile can be stored at $-20{ }^{\circ} \mathrm{C}$ for a week but should preferably be used within a day after alkylation to prevent products from degrading and to obtain better mass spectra.

? TROUBLESHOOTING

(vii) To prepare G00D assay samples for MALDI detection (thin-layer preparation of matrix and sample), first prepare the matrix as $0.1 \% \alpha$-cyano-hydroxy-cinaminic acid methyl ester in acetone. Store this matrix in a refrigerator at $4{ }^{\circ} \mathrm{C}$.

(viii) Apply $0.2 \mu \mathrm{l}$ of the $0.1 \%$ matrix to the hydrophilic spots on the MTP AnchorChip.

(ix) Spot $0.2 \mu \mathrm{l}$ of the GOOD assay sample onto a matrix spot on the MTP AnchorChip and let it dry at room temperature.

(x) Wash the dry preparation by adding a droplet of $2.5 \mu \mathrm{ddd}_{2} \mathrm{O}$. Remove the droplet by aspiration with a pipette after about 20-30 s. Let the sample dry for about $10 \mathrm{~min}$.

(xi) Before MALDI analysis, blow air onto the target plate to avoid contamination of the ionization chamber of the mass spectrometer.

(B) Purification with Oasis microelution plates $\bigcirc$ TIMING 30 min

(i) Wet Oasis resins in the 0asis 96 -well plates with $200 \mu \mathrm{l}$ methanol, apply a vacuum and remove methanol with $200 \mu \mathrm{l}$ $\mathrm{ddH}_{2} \mathrm{O}$, applying the vacuum again.

(ii) Add $\mathrm{ddH}_{2} \mathrm{O}$ to the primer-extension sample to a final volume of $50 \mu \mathrm{l}$, load the sample onto the Oasis resins and apply a vacuum.

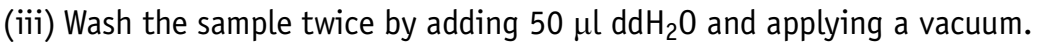

(iv) Elute samples with $50 \mu \mathrm{l}$ methanol, applying a vacuum. Sample the eluates using 96 -well plates (Eppendorf).

(v) Lyophilize the pooled eluates in a SpeedVac Plus SC 210A (Savant).

(vi) Resuspend the dried eluates in $10 \mu \mathrm{lddH_{2 }} 0$.

(vii) To recover the Oasis resins for further experiments, wash them by adding $50 \mu \mathrm{l}$ methanol and applying a vacuum. These resins can be used at least three times, which lowers the cost of the purification procedure.

$\triangle$ CRITICAL STEP The Oasis resins can be used at least three times, but with continued use their performance decreases considerably ${ }^{9}$.

? TROUBLESHOOTING

(viii) To prepare primer-extension products purified as described in Steps $6 \mathrm{~B}(\mathrm{i}$-vii), first prepare $50 \mathrm{mM}$ 3-hydroxypicolionic acid in $3.3 \mathrm{mM}$ ammonium citrate in $\mathrm{ddH}_{2} \mathrm{O}$. The matrix should be stored in a refrigerator at $4{ }^{\circ} \mathrm{C}$ for later use.

(ix) Dispense $0.2 \mu \mathrm{l}$ of the solution prepared in Step 6B(viii) onto the hydrophilic 'anchors' of the target.

(x) Immediately add $0.2 \mu \mathrm{l}$ of the purified primer-extension sample solution and let the droplet dry (about $15 \mathrm{~min}$ ).

(xi) Before MALDI analysis, blow a stream of air onto the target plate to avoid contamination of the ionization chamber of the mass spectrometer.

? TROUBLESHOOTING

NATURE PROTOCOLS | VOL.1 N0.4 | 2006 | 1767 


\section{(C) Microarray purification and sample preparation on the same device TIMING $\mathbf{3 0}$ min}

(i) Prepare $120 \mathrm{mM}$ 3-hydroxypicolionic acid in $15 \mathrm{mM}$ ammonium citrate in $\mathrm{ddH}_{2} 0$. The matrix should be stored in a refrigerator for later use.

(ii) Pattern the slides using a thin marker to prevent spreading of sample solution. For example, plot a quadratic raster with lines at distances of $5 \mathrm{~mm}$ onto the slide.

(iii) Spot $4 \mathrm{nl}$ of crude primer-extension reaction solution onto gold microscope slides coated with PAMAM dendrimer using an nanodispensing robot (e.g., the SciFlex arrayer). For example, the spots can be 'printed', conservatively, at distances of about $5 \mathrm{~mm}$; higher density is possible. Let the droplets dry at room temperature (about $10 \mathrm{~min}$ ).

(iv) Dip the slides, with random orientation, twice in a tub containing $\mathrm{ddH}_{2} \mathrm{O}$.

(v) Dry the slides under a stream of air.

(vi) Spot $4 \mathrm{nl}$ of $120 \mathrm{mM}$ 3-hydroxypicolionic acid in $15 \mathrm{mM}$ ammonium citrate in $\mathrm{ddH}_{2} \mathrm{O}$ onto the slide and let the droplet dry at room temperature (about $10 \mathrm{~min}$ ).

(vii) Clamp the slides in an appropriate slide adapter that fits the geometry of the mass spectrometer used.

(viii) Before MALDI analysis, blow a stream of air over the slides to avoid contamination of the ionization chamber of the mass spectrometer.

$\triangle$ CRITICAL STEP Store the slides coated with PAMAM dendrimer in vacuum. After opening the slide packages use slides immediately or at least in a few days.

? TROUBLESHOOTING

\section{Mass spectrometry TIMING Depends on the number of samples; about $5 \mathrm{~s}$ per sample}

8| Record spectra for products of the G00D assay (option A) in the negative-ion linear time-of-flight mode of a mass spectrometer. Typical acceleration potentials are $18 \mathrm{kV}$. For delayed extraction, the extraction delay is set to $200 \mathrm{~ns}$. On average, ten laser shots are accumulated five times at different positions on the cocrystal of matrix and modified DNA product. The observation window is about $1,000-2,000 \mathrm{~m} \mathrm{z}^{-1}$.

Mass spectra for primer extension products purified by the other two methods (options $B$ and $C$ ) are recorded in positive-ion linear time-of-flight mode using the same acceleration potentials with opposite polarity and same delayed extraction values as for the GOOD assay. On average, five laser shots are accumulated twice per spectrum. The observation window is about $5,000-8,000 \mathrm{~m} \mathrm{z}^{-1}$. The mass accuracy and resolution are lower than for the small products of the G00D assay and can sometimes lead to mass shifts of 'several tens of Daltons'. That can be attributed mainly to the comparably uneven height of 3-hydroxypicolinic acid crystals and the comparably large analyte ions.

9| Process and evaluate spectra using Xmass software (an integral part of the Biflex mass spectrometer). Complete acquisition parameters can be obtained on request; however, in practice, users must optimize these parameters themselves.

\section{Washing MALDI targets TIMING 30 min}

10| Microscope slides cannot be reused. The MTP AnchorChip 400/384 TF targets, however, can be washed and reused at least 50 times. We recommend the following procedure. First, prepare a washing solution containing $30 \%$ isopropanol and $1 \%$ acetic acid in $\mathrm{ddH}_{2} \mathrm{O}$.

11) Rinse the targets first with $\mathrm{ddH}_{2} \mathrm{O}$ and then with acetone.

12| Sonicate the targets in the washing solution for $5 \mathrm{~min}$.

13| Rinse the target with $\mathrm{ddH}_{2} \mathrm{O}$ and then with acetone.

14| Let the target dry before use.

\section{TIMING}

PCR: $2 \mathrm{~h}$

Antarctic phosphatase digestion: $45 \mathrm{~min}$

Primer extension: $2 \mathrm{~h}$

Phosphodiesterase digestion: $100 \mathrm{~min}$

Alkylation: $45 \mathrm{~min}$

Oasis purification: $30 \mathrm{~min}$

Sample preparation of G00D assay or Oasis-purified SNP products for MALDI detection: 30 min

Microarray purification and MALDI preparation on the same device: 30 min 
TABLE 4 | Troubleshooting table.

Problem

insufficient product, as assessed by gel electrophoresis

Antarctic phosphatase digestion: hydrolysis of dNTPs inefficient due to (rare) failure of the enzyme used (usually not critical)

Primer extension: no primer-extension products by mass spectrometry

GOOD assay; phosphodiesterase digestion: additional, larger products because of inefficient digestion, or no products because of very low efficiency

Oasis purification: no products or small amount of products

Microarray purification: no products or small amounts of products

G00D assay; alkylation: no products or no efficient phase separation

Mass spectrometry: no signal

\section{Solution}

Optimization of PCR should be done as recommended by the DNA polymerase supplier. In general, optimization of annealing temperatures and magnesium concentration must be done to resolve problems. Sequence should be checked for duplications in the template genome. Also, homo- or heterodimer formation should be tested with primer design software. Sometimes it is useful to try different primer pairs. Primers could also be of low quality; check primers by MALDI-mass spectrometry or other analytical tools such as HPLC.

Moreover, we recommend using different DNA polymerases to resolve problems with PCR amplification. Most genotyping failures are due to low quality (fragmentation or false concentration) of template DNA. Check template DNA concentration (e.g., with the PicoGreen double-stranded DNA quantification kit and detection with a spectrofluorometer). Check DNA integrity by agarose, or preferentially, by pulsed-field gel electrophoresis.

The efficiency of dNTP hydrolysis by the enzyme can be checked by thin-layer chromatography of nucleotides. In practice, we have not encountered problems with this step.

Low amount of template due to inefficient PCR: check PCR by agarose-gel electrophoresis.

Bad primer: check primer quality by MALDI-MS or other analytical tools such as HPLC.

Bad polymerase: establish a PCR protocol with the polymerase used for primer extension and use this PCR and agarose-gel electrophoresis as a control for enzyme performance. For example, the extension primer and an appropriate PCR primer for the first step can be used to test this.

Bad reagents (e.g., $\alpha$-S-ddNTPs): replace all reagents.

Primer sequence unsuitable (due to consecutive cytosine bases): use primer in the opposite direction.

Bad enzyme: check phosphodiesterase II by adding a confirmed oligonucleotide containing phosphorothioates at the $3^{\prime}$ end, such as extension primers for SNPs; product mass should be detectable after alkylation if the enzyme works well.

Use purified oligonucleotides and oligonucleotides diluted in reaction solution to prove functionality of the plate. Check solutions and vacuum. Consult the manual for the Oasis well plates.

Use purified oligonucleotides and oligonucleotides diluted in reaction solution to prove functionality of surface of slides, which can degrade once slide packages are opened.

Old iodomethane: check quality of iodomethane; brown solution indicates degradation.

Reaction does not work well: check methylation reaction by a small oligonucleotide (four to six nucleotides containing phosphorothioate linkages and phosphate linkage). The methylated product should be observable in the mass spectrometer as GOOD assay products.

Check functionality of the mass spectrometer; if good, check MALDI sample preparation. Test matrix solution and/or surface with 'confirmed' oligonucleotides. Perhaps new matrix must be prepared or the surface must be cleaned again more thoroughly. 


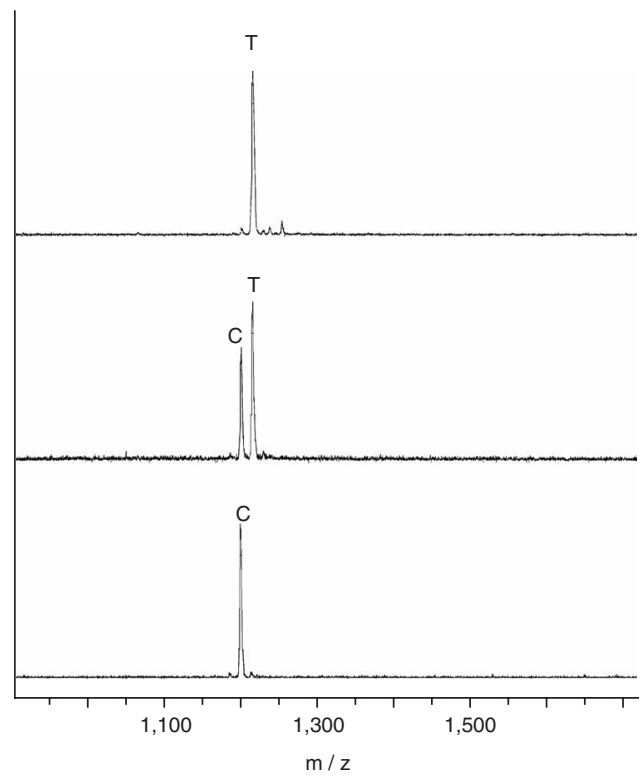

Figure 3 | MALDI mass spectra of the G00D assay products of SNP rs607759, detected in negative-ion mode. The two products of this assay are $\mathrm{G}_{\mathrm{pt}} \mathrm{AC}_{\mathrm{pt}} \mathrm{C}$ $\left(M_{r}, 1,201\right)$ and $\mathrm{G}_{\mathrm{pt}} \mathrm{AC}_{\mathrm{pt}} \mathrm{T}\left(M_{\mathrm{r}}, 1,216\right)$, where the subscripted 'pt' indicates phosphorothioate linkages, which are methylated in the alkylation step of the G00D assay. Top, analysis of a DNA homozygous for I; middle, analysis of heterozygous DNA; bottom, spectrum of DNA homozygous for C. All products can be easily assigned and resolved from each other.

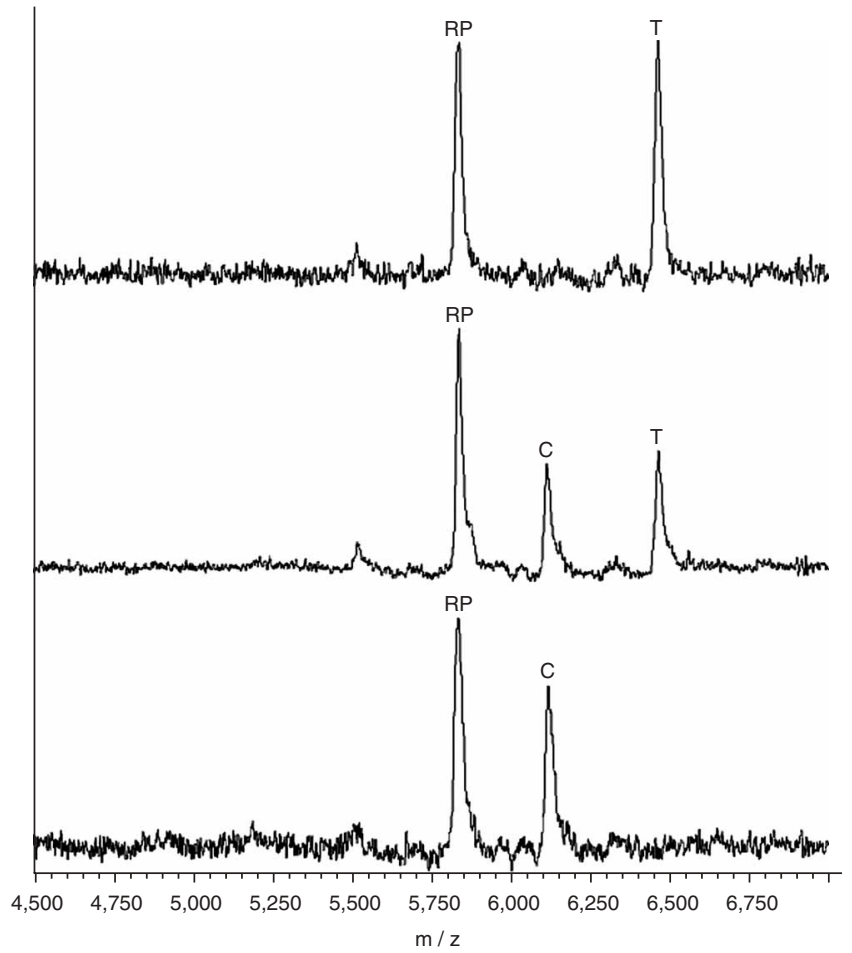

Figure 4 | MALDI mass spectra of unmodified, purified primer-extension products for SNP rs607759, detected in positive-ion mode. The products of the primer-extension reaction, allele $C\left(5^{\prime}\right.$-AATTGAATGGCTCTAGGACC- $\left.3^{\prime} ; M_{\mathrm{r}}, 6,124\right)$ and allele T (5'-AATTGAATGGCTCTAGGACTG-3'; $\left.M_{r}, 6,468\right)$, can be distinguished, and genotypes are assigned according to their masses. The spectra here derive from the microarray approach. Similar spectra are obtained by the conventional dried-droplet method on MALDI targets. RP, residual extension primer (5'-AATTGAATGGCTCTAGGAC-3'; $M_{r}, 5,840$ ).

\section{ANTICIPATED RESULTS}

Anticipated results for GOOD assays of SNP rs607759 by negative-ion mode detection 7,9 are presented in Figure 3. For the same SNP, exemplary results of unmodified primer-extension products purified by either method are presented in Figure 4.

The time-consuming steps are the PCR and the primer-extension reactions. New thermocyclers could complete 40 thermocycles in about $20 \mathrm{~min}$. To avoid potential problems in high-throughput application of thermocyclers resulting from the position of the sample in the plate (the 'edge effect'), we recommend preparing two plates for each set of 384 samples: first, a 'normal' plate, and second, a 'reorganized' plate in which the samples at the periphery of the normal plate are moved to the center.

For medium- to high-throughput applications, the raw data from mass spectrometry are automatically analyzed by software tailored to SNP genotyping, such as the software packages developed by Sequenom or the GenoTools SNP manager developed by Bruker Daltonics ${ }^{19}$. We use the GenoTools SNP manager, which can be adapted for many mass spectrometry SNP-genotyping assays. In general, it analyzes (depending on the parameters defined) spectra quality and genotypes. Although the software for automated SNP 'allele scoring' is steadily improving, particularly for spectra of medium quality or lower, we recommend additional human visual inspection to produce reliable results. In general, the success rates of the SNP assays are over $90 \%$. Repetition of the mass spectrometry analysis could be done to improve the success rate.

The data from SNP-scoring software can be easily exported for further genetic analysis, such as the Hardy-Weinberg check (e.g., with HelixTree (http://www.goldenhelix.com/pharmhelixtreefeatures.html)), tests for mendelian inheritance (e.g., with Pedcheck (http://watson.hgen.pitt.edu/register/)) and many programs for genotype-haplotype and linkageassociation analyses. Many useful links to those genetic analysis software packages can be found on the following website: http://linkage.rockefeller.edu/. In general, in our experience the accuracy of genotype data obtained by our assays is over $99 \%$. 
ACKNOWLEDGMENTS We thank K.-D. Klöppel, K. Neff, P. Kepper, A. Smyra, J. Müller, K. Guse, C. Weidner, A. Dahl and B. M. Kliem for discussions and help. Supported by the European Union (LSHG-CT-2004-503155, MolTools), the German National Genome Research Network (01GR0414), GABI-Génoplante 2 (0313098C) and the Max-Planck Society.

COMPETING INTERESTS STATEMENT The authors declare that they have no competing financial interests.

Published online at http://www.natureprotocols.com

Reprints and permissions information is available online at http://npg.nature.com/ reprintsandpermissions

1. Karas, M. \& Hillenkamp, F. Laser desorption ionization of proteins with molecular masses exceeding 10000 daltons. Anal. Chem. 60, 2299-2303 (1988).

2. Sauer, S. et al. Miniaturization in functional genomics and proteomics. Nat. Rev. Genet. 6, 465-476 (2005).

3. Sauer, S. Typing of single nucleotide polymorphisms by MALDI mass spectrometry: principles and diagnostic applications. Clin. Chim. Acta 363, 95-105 (2006).

4. Brookes, A.J. The essence of SNPs. Gene 234, 177-186 (1999).

5. Sauer, S. \& Gut, I.G. Genotyping single-nucleotide polymorphisms by matrix-assisted laser-desorption/ionization time-of-flight mass spectrometry. J. Chromatogr. B Analyt. Technol. Biomed. Life Sci. 782, 73-87 (2002).

6. Jurinke, C. et al. A single nucleotide polymorphism based approach for the identification and characterization of gene expression modulation using MassARRAY. Mutat. Res. 573, 83-95 (2005).

7. Sauer, S. et al. A novel procedure for efficient genotyping of single nucleotide polymorphisms. Nucleic Acids Res. 28, e13 (2000).

8. Sauer, S. et al. Full flexibility genotyping of single nucleotide polymorphisms by the GOOD assay. Nucleic Acids Res. 28, e100 (2000).

9. Sauer, S. \& Gut, I.G. Extension of the G00D assay for genotyping single nucleotide polymorphisms by matrix-assisted laser desorption/ionization mass spectrometry. Rapid Commun. Mass Spectrom. 17, 1265-1272 (2003).

10. Sauer, S. et al. Automated solid-phase extraction for purification of single nucleotide polymorphism genotyping products prior to matrix-assisted lase desorption/ionisation time-of-flight mass spectrometric analysis. J. Chromatogr. A. 1049, 9-16 (2004).

11. Kepper, P., Reinhardt, R., Dahl, A., Lehrach, H. \& Sauer, S. Matrix-assisted laser desorption/ionization mass spectrometric analysis of DNA on microarrays. Clin. Chem. 52, 1303-1310 (2006).

12. Tost, J., Schatz, P., Schuster, M., Berlin, K. \& Gut, I.G. Analysis and accurate quantification of $\mathrm{CpG}$ methylation by MALDI mass spectrometry. Nucleic Acids Res. 31, e50 (2003).

13. Ding, C. \& Cantor, C.R. Direct molecular haplotyping of long-range genomic DNA with M1-PCR. Proc. Natl. Acad. Sci. USA 100, 7449-7453 (2003).

14. Burgtorf, C. et al. Clone-based systematic haplotyping (CSH): a procedure for physical haplotyping of whole genomes. Genome Res. 13, 2717-2724 (2003).

15. Tost, J. et al. Molecular haplotyping at high throughput. Nucleic Acids Res. 30, e96 (2002).

16. Sauer, S., Lehrach, H. \& Reinhardt, R. MALDI mass spectrometry analysis of single nucleotide polymorphisms by photocleavage and charge-tagging. Nucleic Acids Res. 30, e63 (2003).

17. Sauer, S. et al. Facile method for automated genotyping of single nucleotide polymorphisms by mass spectrometry. Nucleic Acids Res. 30, e22 (2002).

18. Gilar, M., Belenky, A. \& Wang, B.H. High-throughput biopolymer desalting by solid-phase extraction prior to mass spectrometric analysis. J. Chromatogr. A 921 3-13 (2001).

19. Pusch, W., Kraeuter, K.0., Froehlich, T., Stalgies, Y. \& Kostrzewa, M. Genotools SNP manager: a new software for automated high-throughput MALDI-TOF mass spectrometry SNP genotyping. Biotechniques 30, 210-215 (2001). 